\title{
Kay Waechter, Sicherheit und Freiheit in der Rechtsphilosophie
}

Philipp Siegert

\section{OpenEdition}

Journals

Édition électronique

URL : http://journals.openedition.org/ifha/8671

DOI : 10.4000/ifha.8671

ISSN : 2198-8943

Éditeur

IFRA - Institut franco-allemand (sciences historiques et sociales)

Référence électronique

Philipp Siegert, « Kay Waechter, Sicherheit und Freiheit in der Rechtsphilosophie », Revue de I'IFHA [En ligne], Date de recension, mis en ligne le 26 janvier 2017, consulté le 25 septembre 2020. URL : http:// journals.openedition.org/ifha/8671; DOI : https://doi.org/10.4000/ifha.8671

Ce document a été généré automatiquement le 25 septembre 2020

(CIFHA 


\section{Kay Waechter, Sicherheit und Freiheit in der Rechtsphilosophie}

Philipp Siegert

\section{RÉFÉRENCE}

Kay Waechter, Sicherheit und Freiheit in der Rechtsphilosophie, Tübingen: Mohr Siebeck, 2016, 110 p., $39 €$ 
Dans son livre sur « la sécurité et la liberté en philosophie du droit ", Kay Waechter se propose d'explorer le rôle que joue l'équilibre entre sécurité et liberté (ou la primauté accordée à l'un de ces deux principes) dans l'œuvre de onze philosophes, de Platon à Hegel. Sont abordés, outre ces deux jalons, Aristote, Hobbes, Spinoza, Locke, Christian Wolff, Rousseau, Kant, Fichte et Wilhelm von Humboldt. Tout en s'appuyant sur des sources primaires, l'auteur présente de nombreuses réflexions tirées de ces pensées, reflétant par cette démarche son expérience d'enseignant et de praticien $\mathrm{du}$ droit - K. Waechter est professeur de droit et juge. Cet essai entend montrer «combien la réponse [au dilemme sécurité / liberté] dépend de la théorie ", tout en réfléchissant à la question de

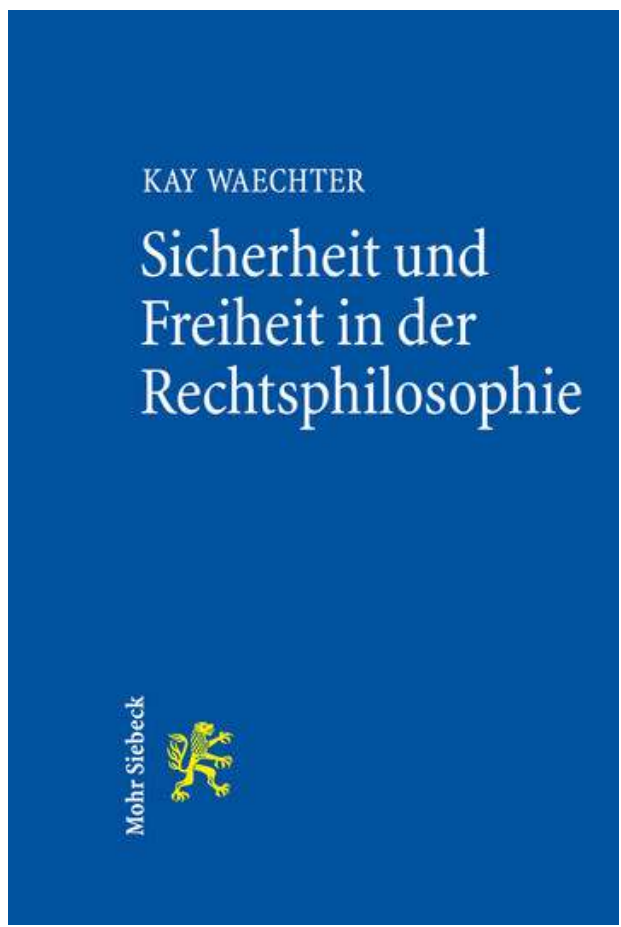
savoir « où, dans le discours sécuritaire, une attention particulière s'impose»(p.1). L'intérêt est donc historique, théorique, mais aussi pratique.

Ce livre d'une centaine de pages a d'abord l'avantage d'être succinct : il offre un accès rapide à la manière dont les philosophes abordés ont pensé le dilemme ancien opposant sécurité et liberté. Le lecteur y trouvera de bons repères, sans être dispensé de lectures plus approfondies pour connaître plus avant les systèmes de pensée dans lesquels ces philosophes situent ce dilemme. Un point fort de cet essai est de s'appuyer sur les sources primaires, et non sur la littérature secondaire (abondante), qu'il évoque peu. Cela lui donne l'avantage de la concision.

Le deuxième point fort de ce livre réside dans les réflexions de l'auteur sur les conséquences pratiques qu'on peut tirer de l'analyse des philosophies étudiées - qui se trouvent déjà en partie chez les auteurs eux-mêmes, comme l'idée de réduire l'interdépendance causée par les dettes des États entre eux pour éviter des crises dans les relations internationales chez Kant (p.59). L'auteur établit de nombreux liens intéressants, ce qui renvoie probablement à sa pratique du droit en tant que juge. En guise d'exemples, on peut citer le contraste qu'il souligne entre la protection de la sphère privée à l'intérieur de la maison chez Fichte et l'état actuel de cette protection (p. 65), ou encore la tentative de Spinoza de garantir la liberté d'expression sans courir le risque de déstabiliser l'État en utilisant cette liberté contre lui, réflexion que s'est appropriée la cour constitutionnelle (Bundesverfassungsgericht) dans une décision de 2010 (p. 40). Cette tendance à établir des liens avec l'actualité est surtout présente dans la conclusion du livre, notamment dans le sous-chapitre intitulé «Pas de devoir de perfectionnement [de l'homme] de la part de l'État dans les conditions du pluralisme » (p. 99-104), particulièrement intéressant.

Quelques - petits - bémols sont toutefois à noter. À plusieurs reprises, le choix des mots semble plus compliqué que nécessaire, parfois à la limite de la confusion. Au début de 
son chapitre sur Fichte, par exemple, l'auteur introduit deux types de contrats sociaux (ou plutôt pré-étatiques), un contrat d'union (Einigungsvertrag) et un contrat d'instauration (Einsetzungsvertrag) sans les expliquer (p.60). Quand il traite, quatre pages plus loin, des étapes de l'établissement de l'État, il utilise les termes de contrat [social] de propriété (Eigentumsvertrag) et contrat [social] de protection (Schutzvertrag) (p. 64). Le lecteur qui n'est pas déjà familier de Fichte peut s'y perdre. De même, la relation entre «société » et « État » reste un peu confuse (en particulier lorsqu'il décrit la police comme « lien » entre l'exécutif et le citoyen, p. 61) ; ce n'est que bien plus tard qu'on lit que «Fichte [aurait] anéanti la séparation entre la société et l'État» (p. 89, dans une comparaison entre Fichte et Hegel).

On pourrait également reprocher à K. Waechter son choix, a priori tout à fait légitime, de se pencher davantage sur certains philosophes que sur d'autres. Ainsi, il consacre moins d'une page et demie à Jean-Jacques Rousseau (p. 49-50), mais un peu plus de sept à Humboldt (p. 72-79) et onze à Platon (p.4-15). En revanche, ce déséquilibre peut augmenter l'intérêt du livre pour un lecteur français, peut-être moins familier de la pensée de Kant ou de Humboldt que de celle de Rousseau.

L'étude de $\mathrm{K}$. Waechter a pour ambition de présenter une première approche de la question de la conciliation entre sécurité et liberté, et cette vue d'ensemble est réussie. Cet essai s'inspire des réflexions de philosophes du droit pour répondre à des questions concrètes en prenant en compte le problème de fond que cela pose: s'il s'agit de décider de mesures individuelles pour contrer un risque, une telle décision doit être guidée par «la sagesse opérationnelle et instrumentale, non par des pensées philosophiques spécifiques » (p. 2). Ce livre ne peut donc présenter qu'un cadre logique permettant de se forger une opinion sur des questions concrètes posant le problème de la conciliation entre sécurité et liberté. C'est la qualité essentielle de ce livre, qui entend moins proposer un panorama des approches qu'une réflexion sur la question de la sécurité et de la liberté.

\section{INDEX}

Thèmes : Histoire des idées

Index chronologique : Ouvrages transpériodiques 